\title{
Downregulation of microRNA-211 is involved in expression of preferentially expressed antigen of melanoma in melanoma cells
}

\author{
EIICHI SAKURAI ${ }^{1,2}$, CHIHAYA MAESAWA $^{1}$, MASAHIKO SHIBAZAKI $^{1}$, SHINJI YASUHIRA $^{1}$, \\ HIROKI OIKAWA $^{3}$, MASAYUKI SATO ${ }^{1}$, KANAKO TSUNODA ${ }^{1,2}$, YUICHI ISHIKAWA ${ }^{1,2}$, \\ AYANO WATANABE $^{1,2}$, KAZUHIRO TAKAHASHI ${ }^{2}$, TOSHIHIDE AKASAKA ${ }^{2}$ and TOMOYUKI MASUDA ${ }^{3}$ \\ Departments of ${ }^{1}$ Tumor Biology, Division of Bioscience, Center for Advanced Medical Science, ${ }^{2}$ Dermatology \\ and ${ }^{3}$ Pathology, School of Medicine, Iwate Medical University, Morioka 020-8505, Japan
}

Received February 22, 2011; Accepted April 19, 2011

DOI: $10.3892 /$ ijo.2011.1084

\begin{abstract}
MicroRNAs (miRNAs) are small non-coding RNAs whose aberrations are involved in the initiation and progression of human cancers. To seek unique miRNAs contributing to melanoma tumorigenesis, we investigated the global miRNA expression profile of 7 melanoma cell lines and 3 primary cultures of neonatal human epidermal melanocytes (NHEMs) using the stem-loop real-time PCR method. We found 7 miRNAs that were commonly downregulated and 18 that were upregulated in all of the melanoma cell lines in comparison with the 3 primary cultures of NHEMs. We focused on one commonly downregulated miRNA (miR-211), and analyzed its relationship to the expression of preferentially expressed antigen of melanoma (PRAME) protein, which is a potential target of miR-211. We found that all melanoma cell lines exhibited marked downregulation of miR-211 and upregulation of PRAME mRNA/ protein expression in comparison with NHEMs $(\mathrm{P}<0.05)$. A significant inverse correlation between miR-211 and PRAME protein expression was found in melanoma cell lines and primary cultures of NHEMs (correlation coefficient of -0.733, $\mathrm{P}<0.05)$. We demonstrated that overexpression of miR-211 induced a reduction of PRAME protein levels, and confirmed the target specificity between miR-211 and PRAME by luciferase reporter assay. These results suggest that downregulation of miR-211 may be partly involved in aberrant expression of the PRAME protein in melanoma cells.
\end{abstract}

\section{Introduction}

microRNAs (miRNAs) are a novel class of conserved 18-25nucleotide RNAs that regulate gene expression in a sequence-specific manner. With $>1000$ already identified

Correspondence to: Dr C. Maesawa, Department of Tumor Biology, Division of Bioscience, Center for Advanced Medical Science, School of Medicine, Iwate Medical University, Uchimaru 19-1, Morioka 020-8505, Iwate, Japan

E-mail: chihaya@iwate-med.ac.jp

Key words: PRAME, microRNA, miR-211, malignant melanoma, cancer-testis antigen (http://microrna.sanger.ac.uk), the human genome may contain up to 1000 miRNAs that play critical roles in cell proliferation, apoptosis and development by negatively regulating the stability or translational efficiency of their target mRNAs $(1,2)$. Aberrant expression of miRNAs has been linked to cancers, and diagnostic/prognostic characteristics of specific cancer types can be distinguished on the basis of their miRNA profiles (3-13). For example, let-7, downregulated in lung cancer $(11,14,15)$, suppresses Ras (15). miR-15 and miR-16, deleted or downregulated in leukemia (16), suppress BCL2 (17) miR-17-5p and miR-20a control the balance of cell death and proliferation driven by the proto-oncogene c-Myc (18). Clear evidence indicates that miRNA polycistron miR-17-92 serves as an oncogene in lymphoma (19) and lung cancer (5). miR-372 and miR-373 are novel oncogenes in testicular germ cell tumors that are involved in the p53 pathway (20). Saito et al (21) demonstrated that epigenetic modification resulting in activation of miR-127 represses translation of BCL6 protein. Most importantly, miRNA expression signatures can predict outcome $(11,14,22)$. These data strongly suggest that miRNAs play an important role in human cancer development.

Although global profiling and functional studies of miRNAs relating to cancer development have been performed in diverse human malignancies $(3-5,7-10,19)$ there are only 3 reports on the miRNA profile of melanoma cells (23-25). Gaur et al (25) examined expression profiles of 241 human miRNAs in normal tissues and the NCI-60 panel of human tumor-derived cell lines including 8 melanoma cell lines. Their agglomerative hierarchical clustering analysis revealed 4 groups among the NCI-60 cell lines comprising hematologic, colon, central nervous system, and melanoma tumor-derived cell lines clustered in a manner that reflected their tissue of origin. They identified 15 melanoma-specific miRNAs in comparison with other types of cancers and normal tissues (although they did not describe whether normal melanocytes had been used as a normal control). Although their results were not wholly identical to those of the other 2 studies, the methodologies (stem-loop real-time PCR method and miRNA microarrays using different platforms) and control samples differed. Moreover, the target genes of miRNAs, which were assigned as melanoma-specific in those reports, have never been functionally examined. To better understand which miRNAs may contribute to melanoma 
development, we investigated the global miRNA expression patterns of 7 melanoma cell lines and 3 primary cultures of neonatal human epidermal melanocytes (NHEM) using the stem-loop real-time PCR method.

We found a unique miRNA (miR-211) that was commonly down-regulated in all of the melanoma cell lines in comparison with primary cultures of NHEM; miR-211 targets preferentially expressed antigen of melanoma (PRAME) protein, which has been well characterized as a cancer-testis antigen through in silico analysis. The gene encoding PRAME was originally cloned by Ikeda et al (26) as part of an investigation into tumor immune surveillance in a patient with a recurrent melanoma. This patient was found to express PRAME, which elicited a cytotoxic T-cell-mediated immune response by autologous lymphocytes (26). The prevalence of PRAME expression is very high in melanomas: $88 \%$ of primary lesions and $95 \%$ of metastases. Gene expression profiling of the different stages of melanoma progression showed that PRAME is expressed in primary melanomas, but not in healthy skin tissue or in benign melanocytic lesions (nevi or moles), suggesting that PRAME expression may be an event in melanocyte transformation (27). We were able to demonstrate an inverse correlation between miR-211 and PRAME protein expression, and obtain in vitro evidence that loss of miR-211 expression is involved in PRAME protein translation in melanoma cells.

\section{Materials and methods}

Cell lines. Four human melanoma cell lines (HMV-I, HMV-II, G-361 and SK-MEL-28) and 3 neonatal human epidermal melanocyte cell lines (NHEM-L, NHEM-M and NHEM-D) were obtained from the Cell Resource Center for Biomedical Research, Tohoku University (Sendai, Japan), and Cascade Biologics Inc. (Portland, OR, USA), respectively. Three melanoma cell lines (MM-EP, MM-RU and MM-WK) were kindly donated by Professor M.C. Mihm (Department of Dermatology, Harvard Medical University, Boston, MA, USA). A melanoma cell line (MM-RU) was established from a metastatic tumor (lymph node), and another cell line (MM-EP) was derived from a recurrent site (28). Remaining 5 lines were established from primary sites. Human embryonic kidney cell line 293 (HEK-293) was obtained from the American Type Culture Collection (Manassas, VA, USA). All cell lines were maintained under the recommended conditions.

Stem-loop real-time PCR for miRNA quantification. We used a TaqMan MicroRNA Assay Human Panel-Early Access Kit (including 157 miRNAs and 3 negative controls) (Applied Biosystems; ABI, Foster City, CA, USA) for quantitative RT-PCR assay. Expression levels of miRNAs were analyzed using an ABI PRISM 7500 instrument (ABI). Briefly, cDNA was made from enriched miRNA in $15 \mu \mathrm{l}$ reaction volumes (10 ng/well) using Multiscribe (MuLV) reverse transcriptase and specific primers for each miRNA. The cycle parameters for the reverse-transcriptase reaction were $16^{\circ} \mathrm{C}$ for $30 \mathrm{~min}$, $42^{\circ} \mathrm{C}$ for $30 \mathrm{~min}, 85^{\circ} \mathrm{C}$ for $5 \mathrm{~min}$ and a hold at $4^{\circ} \mathrm{C}$. The PCR reaction mix consisted of the RT product, TaqMan 2x Universal PCR Master Mix and the appropriate 10x MicroRNA Assay Mix containing primers and a probe for the miRNA of interest. Cycle parameters for the PCR reaction were $95^{\circ} \mathrm{C}$ for $10 \mathrm{~min}$, followed by 50 cycles of a denaturing step at $95^{\circ} \mathrm{C}$ for $15 \mathrm{sec}$ and an annealing/extension step at $60^{\circ} \mathrm{C}$ for $60 \mathrm{sec}$. All reactions were run in tripicate. A U6 primer/probe set was made as follows: U6-360F, TGG AAC GAT ACA GAG AAG ATT AGC A; U6-425R, AAC GCT TCA CGA ATT TGC GT; FAM-CCC CTG CGC AAG GA-MGB. The expression of each miRNA relative to $\mathrm{U} 6$ was determined using the $\Delta \Delta \mathrm{Ct}$ method. The threshold cycle $(\mathrm{Ct})$ is defined as the fractional cycle number at which the fluorescence passes the fixed threshold. Average fold differences were calculated by normalizing the relative expression ( $\Delta \Delta \mathrm{Ct}$ values) according to the User Bulletin $\# 2$ (ABI).

Western blotting. Nuclear protein was extracted using NE-PER Nuclear and Cytoplasmic Reagents (Pierce, Woburn, MA, USA). Lysates were mixed with $6 x$ concentrated loading dye, heated for $5 \mathrm{~min}$ at $95^{\circ} \mathrm{C}$, and subjected to SDS-PAGE on a $7.5 \%$ polyacrylamide gel (Ready Gels J, Bio-Rad, Hercules, CA, USA). The proteins were then transferred to a PVDF membrane (Hybond-P, GE Healthcare, Buckinghamshire, UK) by electroblotting. The primary antibodies against PRAME (E-19) (sc-34512, Santa Cruz Biotechnology, Santa Cruz, CA, USA) and lamin B (M-20) (sc-6217, Santa Cruz Biotechnology) were diluted 1:500 in immunoreaction enhancer solution (Can Get Signal Solution 1; Toyobo Co., Ltd., Osaka, Japan). The membrane was incubated for $1 \mathrm{~h}$ at room temperature. The second antibody, anti-goat IgG (GE Healthcare), was diluted 1:5000 in immunoreaction enhancer solution (Can Get Signal Solution 2, Toyobo). The membrane was incubated for $60 \mathrm{~min}$ at room temperature and washed. Signals were detected with ECL Plus (GE Healthcare) and ChemiDoc XRS (Bio-Rad). The intensity of detected signals was measured by 1-D analysis software (Quantity One, Bio-Rad).

Quantitative real-time RT-PCR. Total RNA was isolated with TRIzol reagent (Invitrogen, Carlsbad, CA, USA). mRNA was reverse-transcribed with a Superscript ${ }^{\mathrm{TM}}$ III First-Strand Synthesis SuperMix and oligo(dT) (Invitrogen) to produce cDNA. For the assay, Custom TaqMan Gene Expression Assays and an ABI PRISM 7500 instrument (ABI) were used. For normalization of the target, glyceraldehyde-3-phosphate dehydrogenase (GAPDH, ABI) was used as an internal control. All reactions were run in triplicate and average fold differences were calculated by normalizing the relative expression $(\Delta \Delta \mathrm{Ct}$ values) as described previously in accordance with the User Bulletin \#2 (ABI).

Transfection with miR-211 precursor molecules. miR-211 precursor molecule and 'negative control 1' precursor miRNAs were purchased from ABI. They were transfected into two melanoma cell lines (G-361 and SK-MEL-28) and HEK-293 at final concentrations of $100 \mathrm{nM}$ each using lipofectamine RNAiMAX (Invitrogen) according to the manufacturer's instructions. One day after transfection, the cells were harvested and PRAME expression was analyzed by Western blotting.

Luciferase assay. Luciferase constructs were made by ligating oligonucleotides containing the wild-type or mutant target site of PRAME into the pMIR-Report ${ }^{\mathrm{TM}}$ vector, as described previously (21). Melanoma cell lines (G-361 and SK-MEL-28) 
Table I. The commonly altered miRNAs in all 7 melanoma cell lines in comparison with 3 primary cultures of NHEM.

Up- or downregulation

Upregulation

Downregulation
Altered miRNAs

miR-17-3p, -19a, -21, -27b, -29b, -30e, -96, -100, -105, -124b, -125b, -126, -147, -182, $-183,-219,-302 b^{*},-373$

miR-150, -155, -186, -191, -197, -211, -223

Relative expression differences $<0.5$ or $>1.5$ were considered to represent a significant difference between two cell lines.

A

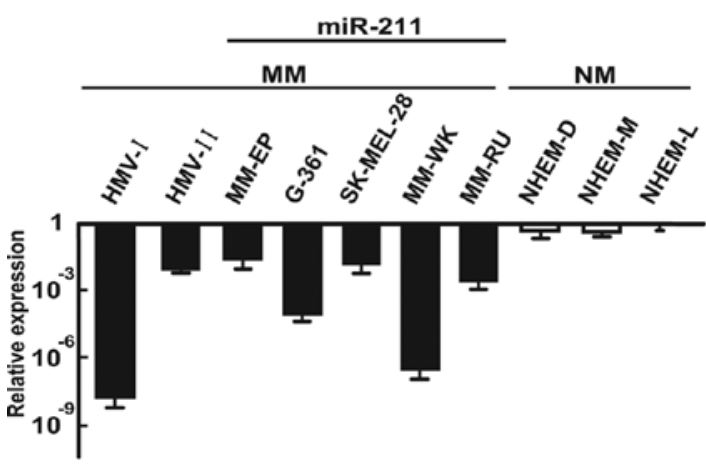

B

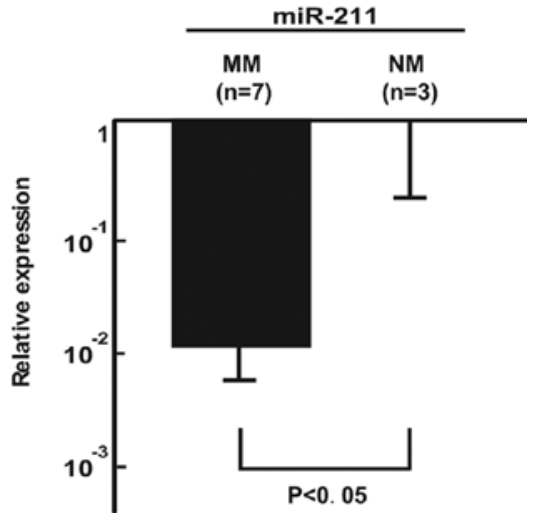

Figure 1. Quantitative expression of miR-211 in melanoma cell lines and primary cultures of NHEM. (A) MiR-211 expression in 7 melanoma cell lines and 3 primary cultures of NHEM. Relative miR-211 expression data were calculated using the $\Delta \Delta \mathrm{CT}$ method with U6 RNA as an endogenous control. Each value represents the relative fold increase $\left(\log 2^{-(\Delta \Delta C t)}\right)$ of miR-211 using the mean value for primary cultured cells (NHEM-L) as the reference sample. All melanoma cell lines exhibited downregulation of miR-211 expression. MM, malignant melanoma. NM, normal melanocyte. (B) Comparison of miR-211 expression between 7 melanoma cell lines and 3 primary cultures of NHEM. The miR-211 expression in melanoma cell lines was significantly downregulated in comparison with NHEM. MM, malignant melanoma. NM, normal melanocyte.

and HEK-293 were transfected with 50 ng of luciferase reporter vector containing a wild-type or mutant target site and $50 \mathrm{ng}$ of the control vector (pMIR-Report containing $\beta$-gal control plasmid) (ABI), using Lipofectamine 2000 (Invitrogen) in 96-well plates. Luciferase assays were performed $24 \mathrm{~h}$ after transfection using the Dual Luciferase Reporter Assay System (Dual-Light System, ABI). Six times assays were performed for each experiment.

Statistical analysis. Data were analyzed by Mann-Whitney U test for non-parametric samples. All data are presented as the mean \pm SE. Spearman's correlation coefficient by rank test (Spearman's rank correlation) was introduced to assess the relationship between miRNA and target protein expression.

\section{Results}

miRNA profiles of melanoma cell lines and primary cultures of NHEM detected by the stem-loop real-time PCR method. To obtain miRNA profiles in our series, we employed the stem-loop real-time PCR method rather than miRNA microarray, because the latter has limited specificity when an oligonucleotide probe is used. We found 18 up- and 7 down-regulated miRNAs that were common to all 7 melanoma cell lines in comparison with the 3 primary cultures of NHEM (Table I). miRNAs that were commonly up- and downregulated in $>5$ cell lines are presented in Table I.

Although there were several interesting miRNAs associated with cancer initiation/progression, we focused on miR-211 on the basis of data from in silico analysis (miRBase, http:// microrna.sanger.ac.uk) because miR-211 targets PRAME protein, which is a well known cancer-testis antigen that shows frequent aberrant expression in melanoma cells.

Relationship between miR-211 and PRAME protein expression in melanoma cell lines and primary cultures of NHEM. Fig. 1A shows miR-211 expression in each melanoma cell line and primary culture of NHEM. miR-211 expression was significantly downregulated in 7 melanoma cell lines in comparison with the 3 primary cultures of NHEM ( $<<0.05$, Fig. 1B). We further examined PRAME mRNA/protein, a potential target of miR-211, using real-time quantitative PCR and Western blot analyses. The real-time quantitative PCR assays demonstrated equal up-regulation of PRAME mRNA in all melanoma cell lines in comparison with primary cultures of NHEM (Fig. 2A). In contrast to the quantitative data for PRAME mRNA, the expression of PRAME protein varied among the melanoma cell lines (Fig. 2B). As expected, endogenous expression of PRAME protein in NHEM was uniformly low, in accord with the expression of its mRNA. Quantitative expression of 
A

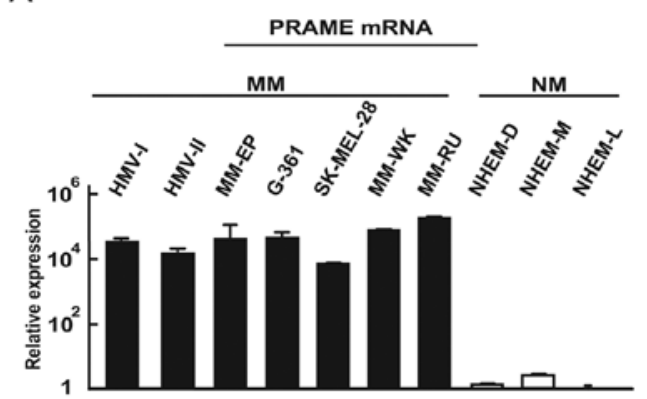

B

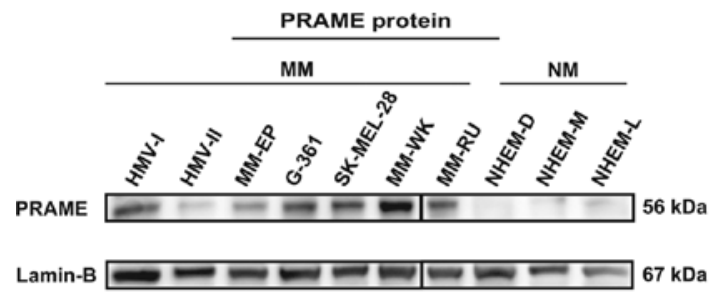

C

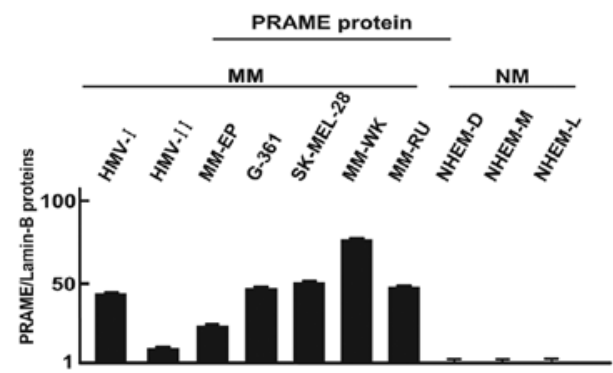

D

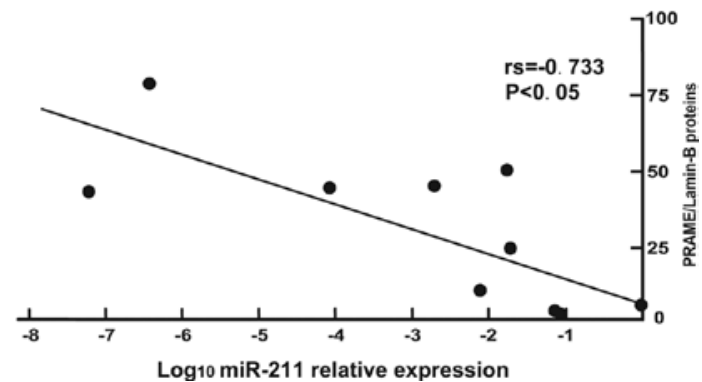

Figure 2. PRAME mRNA and protein expression in melanoma cell lines and primary cultures of NHEM. (A) PRAME mRNA expression in 7 melanoma cell lines and 3 primary cultures of NHEM. Relative PRAME mRNA expression data were calculated using the $\triangle \Delta C T$ method with GAPDH as an endogenous control. Each value represents the relative fold increase $\left(\log 2^{-(\Delta \Delta \mathrm{Ct})}\right)$ of PRAME mRNA using the mean value for primary cultured cells (NHEM-L) as the reference sample. Marked upregulation was observed in all melanoma cell lines in comparison with normal melanocytes. MM, malignant melanoma. NM, normal melanocyte. (B) Western blotting for PRAME protein in 7 melanoma cell lines and 3 primary cultures of NHEM. Twenty micrograms of nuclear extract were applied to each lane. Equal loading was confirmed by blotting of lamin B (Lamin-B). Five melanoma cell lines exhibited dense signals (HMV-I, G-361, SK-MEL-28, MM-WK and MM-RU) and two (HMV-II, MM-EP) showed relatively weak signals. All primary cultures of NHEM showed an endogenous level. MM, malignant melanoma. NM, normal melanocyte. (C) Quantitative data for PRAME protein expression in 7 melanoma cell lines and 3 primary cultures of NHEM. The expression level varied among the melanoma cell lines. MM, malignant melanoma. NM, normal melanocyte. (D) Scatter plots of quantitative values for miR-211 and PRAME protein. Spearman's correlation coefficient by rank (Spearman's rank correlation) was introduced (correlation coefficient value: $r s=-0.7333, \mathrm{p}<0.05)$.
PRAME protein was relatively lower in 2 of the melanoma cell lines (HMV-II and MM-EP) than in the other 5 (HMV-I, G-361, SK-MEL-28, MM-WK and MM-RU) (Fig. 2C). miR-211 expression in these cell lines with relatively weak expression of PRAME protein (HMV-II and MM-EP) was higher than that in the cell lines with relatively strong expression of PRAME protein. A significant inverse correlation between miR-211 and PRAME protein expression was observed in all cell lines examined (Fig. 2D; correlation coefficient value, rs $=-0.7333, \mathrm{P}<0.05$; Spearman's correlation coefficient by rank test).

In silico and quantitative analyses of miRNAs targeting PRAME protein. Furthermore, in silico analysis indicated that 58 miRNAs targeted the PRAME gene. The stem-loop-mediated reverse transcription real-time PCR method allowed us to examine 11 of these 58 miRNAs (including miR-211). The locations of the base-pairing nucleotides within the PRAME 3'-untranslated region (UTR) are shown in Fig. 3A. Two sites matching miR-211 were found within the PRAME 3'-UTR. The 2 binding sites were located close to each other.

The quantitative data indicated that out of the 11 examined miRNAs targeting PRAME protein, only miR-211 was commonly downregulated in all melanoma cell lines as compared with the primary cultures of NHEM (Figs. 1A and 3B).

Induction studies of miR-211 precursor molecule. Next, we examined whether miR-211 induction was able to repress PRAME protein expression. Because we had preliminarily confirmed that miR-211 precursor molecules had relatively inferior transfection efficiency in melanoma cell lines, we also examined HEK-293 cells. Overexpression of miR-211 precursor molecules induced a reduction of the PRAME protein level in all cell lines in comparison with the negative control (Fig. 4A and B). However, the PRAME mRNA showed no marked alteration in any of the cell lines (Fig. 4C). This means that miR-211 repression occurs mainly at the translational level.

To further confirm the target specificity of miR-211 for PRAME, we performed a luciferase reporter assay with a vector containing the putative PRAME 3'-UTR target sites downstream of a luciferase reporter gene. Base pairing between miR-211 and the wild-type (WT) or mutant (M1 and M2) putative target site in the 3'-UTR of PRAME mRNA is shown in Fig. 5A. A M3 luciferase vector, which contained both M1 and M2, was also generated. Luciferase activity of cell lines transfected with PRAME-WT was significantly lower than those transfected with the PRAME-M vectors (M1, M2 and M3) $(\mathrm{P}<0.05)$ (Fig. 5B). Luciferase activity was accelerated in all cell lines transfected with the M3 vector in comparison with those transfected with the single-mutation vectors (M1 and M2) (Fig. 5B). These data suggest that miR-211 is able to act as a translational repressor for PRAME protein in melanoma cells as well as HEK-293 cells.

\section{Discussion}

In this study we were able to demonstrate for the first time an inverse relationship between miR-211 and aberrant expression of PRAME protein, a cancer-testis antigen, in malignant melanoma cell lines. Although a previous study has documented 
A
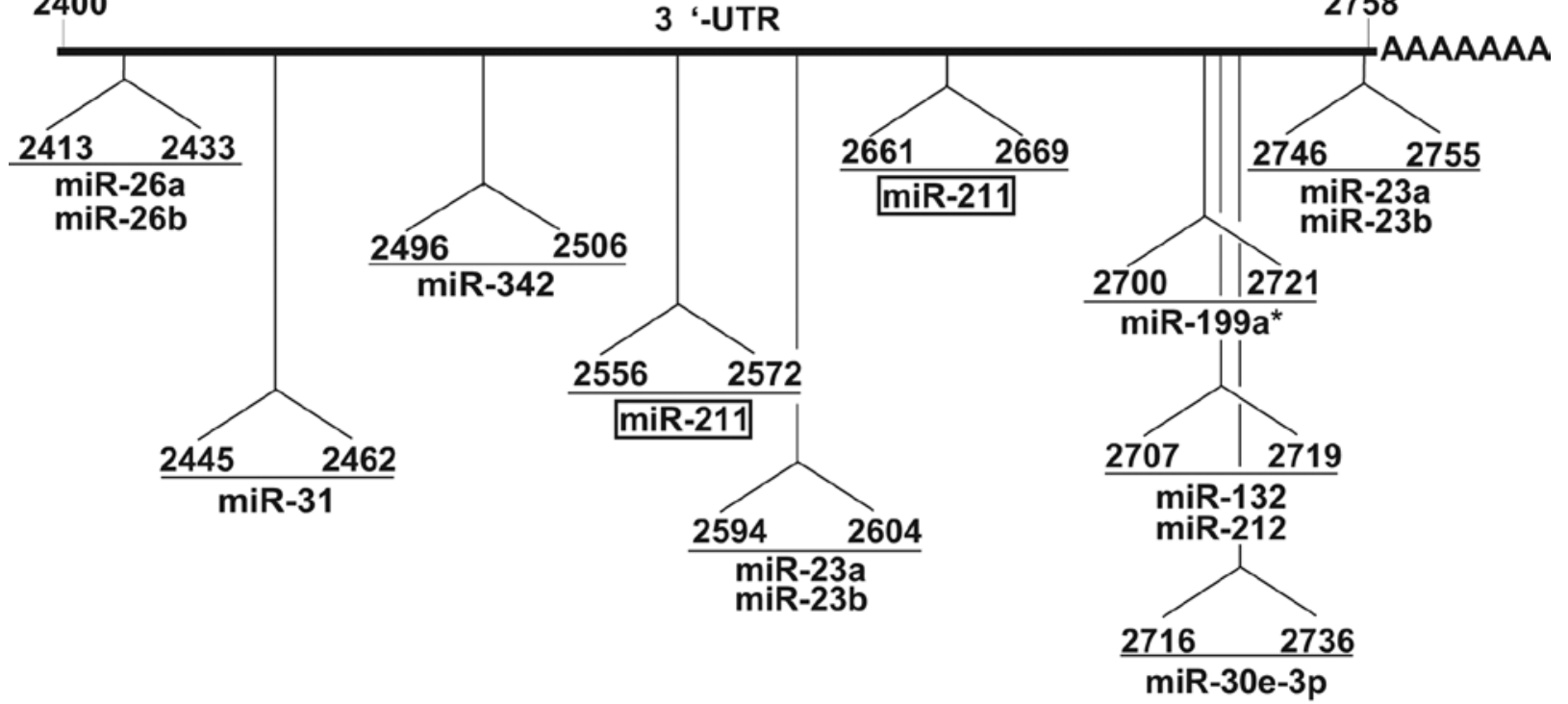

B
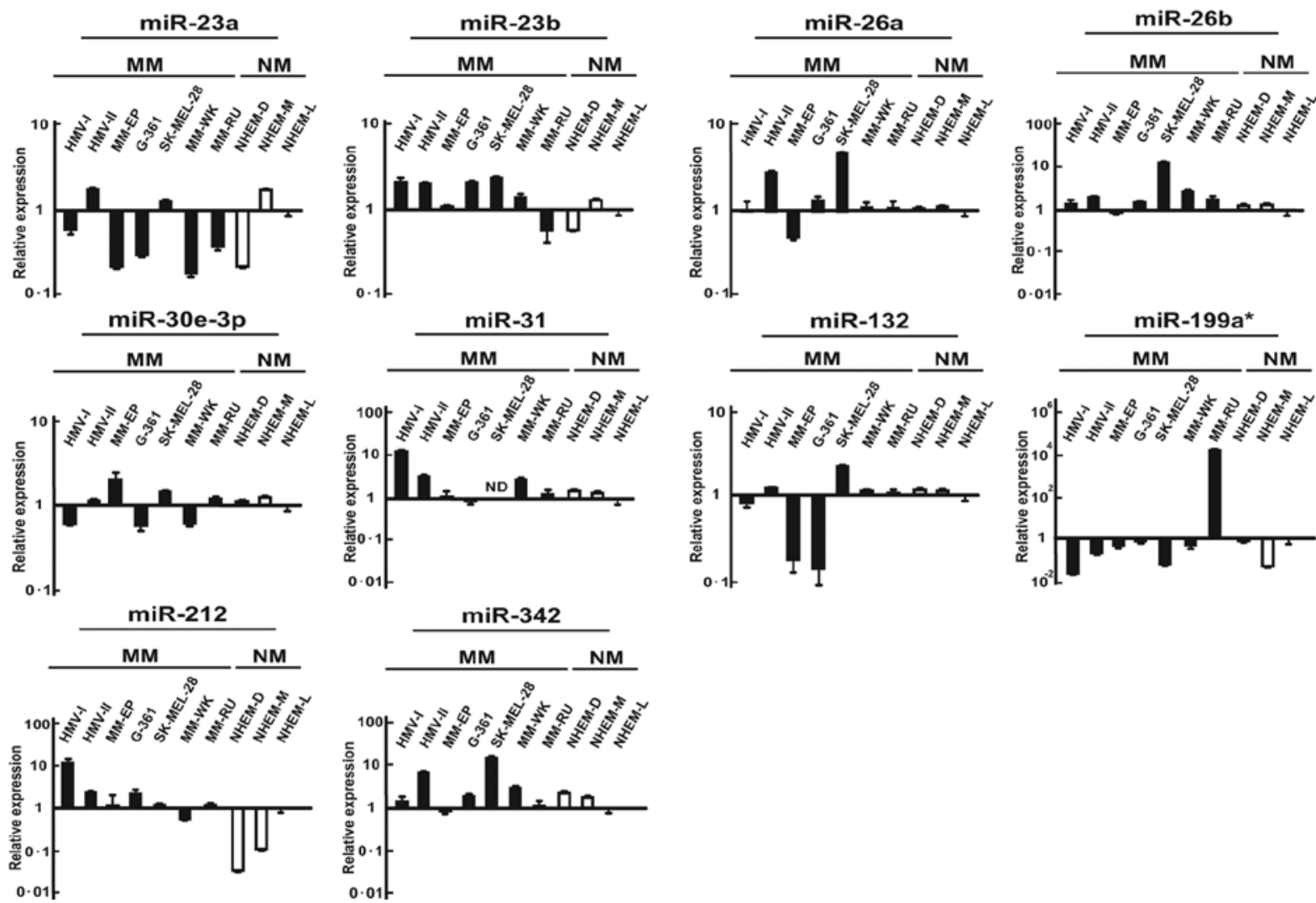

Figure 3. Location of base pairing site of miRNAs targeting PRAME mRNA and their quantitative data in melanoma cell lines and primary cultures of NHEM. (A) Schematic representation of the locations of the base-pairing nucleotides of 11 miRNAs within the PRAME 3'-UTR. Numbers are the nucleotide positions for PRAME mRNA. MiR-211 (square circled), which was the focus of study, has two binding sites close to each other. (B) Expression of miRNAs except for miR-211 in 7 melanoma cell lines and 3 primary cultures of NHEM. Analysis of relative miRNA expression data was performed using the $\Delta \Delta C T$ method with U6 RNA as an endogenous control. Each value represents the relative fold increase $\left(\log 2^{-(\Delta \Delta C t)}\right)$ of each miRNA obtained using the mean value for primary culture cells (NHEM-L) as the reference sample. In contrast to miR-211, quantitative results varied among miRNAs. MM, malignant melanoma. NM, normal melanocyte.

that miR-211 is a unique miRNA that reflects the tissue of origin of melanocytic tumors (25), the significance of its downregulation has never been functionally examined in melanoma cells. The aim of that study was to identify clusters according to tumor cell types using multiscale bootstrap analysis. Among 241 examined miRNAs, 42 were available for clustering of 4 
tumor types. However, among these 42 miRNAs, miR-211 and miR-375 were excluded from clustering analysis because their individual removal from the multiscale bootstrap analysis had no effect. In fact, we have also examined miR-211 expression in a wide variety of human malignant cell lines, and observed frequent loss of miR-211 in several cancer types (data not shown). These data suggest that downregulation of miR-211 might be a relatively common event in human malignant cell lines.

High prevalence of aberrant PRAME expression has been reported not only in malignant melanoma but also in non-small cell lung carcinoma (26), breast carcinoma (26), renal cell carcinoma (29), head and neck cancers (26), Hodgkin's lymphoma (30), sarcomas (26), Wilms' tumor (31) and medulloblastomas (approximately 30-70\%) (32). In contrast to other types of cancer-testis antigen, PRAME is also expressed in some normal tissues other than testis (bone marrow/CD34-positive cells, ovary and adrenal gland) (26). The high activation of cancertestis antigen in testis may be explained by genome-wide DNA demethylation, which occurs in male germline cells. Although the cause of aberrant PRAME expression in malignancies has been unclear, it was recently reported that DNA hypomethylation of PRAME promoter is responsible for its aberrant overexpression in human acute myeloid leukemia (AML) and a few epithelial cell lines (33). Although no systemic epigenetic studies (DNA methylation and/or chromatin methylation/acetylation analyses) have ever examined melanomas and NHEM, one report has described that a demethylating agent, 5-aza-2'-deoxycytidine, was able to induce PRAME mRNA expression in PRAMEnegative primary cultures derived from a primary melanoma (34). These data led us to speculate that DNA hypomethylation at the promoter region is related to aberrant expression of PRAME in melanoma cells. Before the present study, we preliminarily examined the DNA methylation status in melanoma cell lines and primary cultures of NHEM. Hypermethylation at the PRAME promoter was observed in 3 primary cultures of NHEM, and the methylation status of melanoma cells did not always correlate with their expression of PRAME protein (data not shown). In addition, even though two melanoma cell lines (HMV-II and MM-EP) overexpressed PRAME mRNA, PRAME protein expression was relatively weak in comparison with other melanoma cell lines. These findings suggested that PRAME protein expression might be under post-transcriptional regulation in melanoma cells. Considering these data, we hypothesized that aberrant PRAME protein expression might be blocked both epigenetically and by expression of miRNA.

PRAMEexpression is implicated in oncogenic transformation during melanoma tumorigenesis, rather than merely being a marker of progressive disease, because healthy skin tissues or benign melanocytic lesions are negative for PRAME, in contrast to the high incidence of PRAME-positive melanomas (35). However, a novel report has indicated that PRAME is a dominant repressor of retinoic acid (RA) receptor (RAR) signaling (35). RAR signaling plays an essential role in development, cell fate determination, and tissue homeostasis. Inactivation of RA-RAR signaling is beneficial to cancer cells because it induces transcriptional activation of related genes, cell cycle arrest and/or differentiation. The PRAME protein binds to the RAR through nuclear receptor boxes and modulates the activity of the RA-RAR signaling pathway (35). Ectopic expression of
PRAME in RA-sensitive cells was shown to confer resistance to RA-induced growth arrest, differentiation, and apoptosis (35). Moreover, knockdown of PRAME by RNA interference in melanoma cells, which are invariably RA-resistant, restored their sensitivity to RA and induction of the target genes RAR $\beta$ and $\mathrm{p} 21$. In addition, melanoma xenografts with PRAME knockdown showed significantly reduced growth after treatment with RA, compared with xenografts without PRAME knockdown (35). Thus, PRAME-RA-RAR could be a potential target for therapy of malignant melanomas.

In anticipation of an anti-tumor effect of RA, a few phase II trials of RA plus interferon therapy have been conducted in patients with advanced melanoma as well as other tumor types $(36,37)$, although the response rates were unsatisfactory. The high prevalence of aberrant PRAME expression in melanomas might partially affect the efficacy of RA. Epping and Bernards (38) indicated two strategies for PRAME targeting therapy. First, immunotherapy could be designed to boost the T-cell-mediated immune response to PRAME-positive tumor cells. However, whether patients can be stimulated to consistently mount a robust immunologic response to this antigen has not yet been established. The first clinical study targeting PRAME used autologous AML-dendritic cells, which were injected into patients with AML (39). This resulted in an increase of T-cell numbers specifically recognizing a PRAME-derived peptide (39). Alternatively, small molecule-based strategies to target PRAME in cancer can be envisioned. However, PRAME has not been shown to possess intrinsic enzymatic activity that could be targeted by small-molecule drugs. In addition, the small hydrophobic $\alpha$-helical interaction surface between PRAME and RAR is a difficult target for drug development. Although several problematic issues remain in the development of PRAME-targeting therapies for malignant melanoma, at least downregulation of PRAME protein would be a key step in the development of RA-based combination therapies. Modulation of miR-211 expression may play an important role in the development of such therapies.

\section{Acknowledgements}

This work was supported in part by Grants-in-Aid for Scientific Research (30509013, 10326647, 10326647 and 16791346), and the MIAST (Medical Innovation by Advanced Science and Technology; 2010-2014) project, Grant-in-Aid for Strategic Medical Science Research Center from the Ministry of Education, Culture, Sports, Science and Technology of Japan.

\section{References}

1. He L and Hannon GJ: MicroRNAs: small RNAs with a big role in gene regulation. Nat Rev Genet 5: 522-531, 2004.

2. Meltzer PS: Cancer genomics: small RNAs with big impacts. Nature 435: 745-746, 2005.

3. Calin GA, Liu CG, Sevignani C, et al: MicroRNA profiling reveals distinct signatures in B cell chronic lymphocytic leukemias. Proc Natl Acad Sci USA 101: 11755-11760, 2004.

4. Ciafre SA, Galardi S, Mangiola A, et al: Extensive modulation of a set of microRNAs in primary glioblastoma. Biochem Biophys Res Commun 334: 1351-1358, 2005.

5. Hayashita $\mathrm{Y}$, Osada $\mathrm{H}$, Tatematsu $\mathrm{Y}$, et al: A polycistronic microRNA cluster, miR-17-92, is overexpressed in human lung cancers and enhances cell proliferation. Cancer Res 65: 9628-9632, 2005. 
6. He H, Jazdzewski K, Li W, et al: The role of microRNA genes in papillary thyroid carcinoma. Proc Natl Acad Sci USA 102: 19075-19080, 2005.

7. Iorio MV, Ferracin M, Liu CG, et al: MicroRNA gene expression deregulation in human breast cancer. Cancer Res 65: 7065-7070, 2005.

8. Jiang J,Lee EJ, Gusev Y and Schmittgen TD: Real-time expression profiling of microRNA precursors in human cancer cell lines. Nucleic Acids Res 33: 5394-5403, 2005.

9. Lu J, Getz G, Miska EA, et al: MicroRNA expression profiles classify human cancers. Nature 435: 834-838, 2005.

10. Volinia S, Calin GA, Liu CG, et al: A microRNA expression signature of human solid tumors defines cancer gene targets. Proc Natl Acad Sci USA 103: 2257-2261, 2006.

11. Yanaihara N, Caplen N, Bowman E, et al: Unique microRNA molecular profiles in lung cancer diagnosis and prognosis. Cancer Cell 9: 189-198, 2006.

12. Bottoni A, Zatelli MC, Ferracin M, et al: Identification of differentially expressed microRNAs by microarray: a possible role for microRNA genes in pituitary adenomas. J Cell Physiol 210: 370-377, 2007.

13. Lee EJ, Gusev Y, Jiang J, et al: Expression profiling identifies microRNA signature in pancreatic cancer. Int J Cancer 120 1046-1054, 2007.

14. Takamizawa J, Konishi H, Yanagisawa K, et al: Reduced expression of the let-7 microRNAs in human lung cancers in association with shortened postoperative survival. Cancer Res 64: 3753-3756, 2004.

15. Johnson SM, Grosshans H, Shingara J, et al: RAS is regulated by the let-7 microRNA family. Cell 120: 635-647, 2005.

16. Calin GA, Dumitru CD, Shimizu M, et al: Frequent deletions and down-regulation of micro-RNA genes miR15 and miR16 at $13 q 14$ in chronic lymphocytic leukemia. Proc Natl Acad Sci USA 99: 15524-15529, 2002.

17. Cimmino A, Calin GA, Fabbri M, et al: miR-15 and miR-16 induce apoptosis by targeting BCL2. Proc Natl Acad Sci USA 102: 13944-13949, 2005.

18. O'Donnell KA, Wentzel EA, Zeller KI, Dang CV and Mendell JT: c-Myc-regulated microRNAs modulate E2F1 expression. Nature 435: 839-843, 2005.

19. He L, Thomson JM, Hemann MT, et al: A microRNA polycistron as a potential human oncogene. Nature 435: 828-833, 2005.

20. Voorhoeve PM, le Sage C, Schrier M, et al: A genetic screen implicates miRNA-372 and miRNA-373 as oncogenes in testicular germ cell tumors. Cell 124: 1169-1181, 2006.

21. Saito Y, Liang G, Egger G, et al: Specific activation of microRNA-127 with downregulation of the proto-oncogene BCL6 by chromatin-modifying drugs in human cancer cells. Cancer Cell 9: 435-443, 2006.

22. Calin GA, Ferracin M, Cimmino A, et al: A MicroRNA signature associated with prognosis and progression in chronic lymphocytic leukemia. N Engl J Med 353: 1793-1801, 2005.

23. Zhang L, Huang J, Yang N, et al: microR NAs exhibit high frequency genomic alterations in human cancer. Proc Natl Acad Sci USA 103: 9136-9141, 2006.

24. Blower PE, Verducci JS, Lin S, et al: MicroRNA expression profiles for the NCI-60 cancer cell panel. Mol Cancer Ther 6: $1483-1491,2007$.
25. Gaur A, Jewell DA, Liang Y, et al: Characterization of microRNA expression levels and their biological correlates in human cancer cell lines. Cancer Res 67: 2456-2468, 2007.

26. Ikeda H, Lethe B, Lehmann F, et al: Characterization of an antigen that is recognized on a melanoma showing partial HLA loss by CTL expressing an NK inhibitory receptor. Immunity 6 : 199-208, 1997.

27. Haqq C, Nosrati M, Sudilovsky D, et al: The gene expression signatures of melanoma progression. Proc Natl Acad Sci USA 102: 6092-6097, 2005.

28. Hoashi T, Kadono T, Kikuchi K, Etoh T and Tamaki K: Differential growth regulation in human melanoma cell lines by TIMP-1 and TIMP-2. Biochem Biophys Res Commun 288: 371-379, 2001.

29. Neumann E, Engelsberg A, Decker J, et al: Heterogeneous expression of the tumor-associated antigens RAGE-1, PRAME, and glycoprotein 75 in human renal cell carcinoma: candidates for T-cell-based immunotherapies? Cancer Res 58: 4090-4095, 1998.

30. Willenbrock K, Kuppers R, Renne C, et al: Common features and differences in the transcriptome of large cell anaplastic lymphoma and classical Hodgkin's lymphoma. Haematologica 91: 596-604, 2006.

31. Li CM, Guo M, Borczuk A, et al: Gene expression in Wilms' tumor mimics the earliest committed stage in the metanephric mesenchymal-epithelial transition. Am J Pathol 160: 2181-2190, 2002.

32. Boon K,Edwards JB, Siu IM, et al: Comparison of medulloblastoma and normal neural transcriptomes identifies a restricted set of activated genes. Oncogene 22: 7687-7694, 2003.

33. Schenk T, Stengel S, Goellner S, Steinbach D and Saluz HP: Hypomethylation of PRAME is responsible for its aberrant overexpression in human malignancies. Genes Chromosomes Cancer 46: 796-804, 2007.

34. Sigalotti L, Fratta E, Coral S, et al: Intratumor heterogeneity of cancer/testis antigens expression in human cutaneous melanoma is methylation-regulated and functionally reverted by 5 -aza-2'deoxycytidine. Cancer Res 64: 9167-9171, 2004.

35. Epping MT, Wang L, Edel MJ, Carlee L, Hernandez M and Bernards R: The human tumor antigen PRAME is a dominant repressor of retinoic acid receptor signaling. Cell 122: 835-847, 2005.

36. Rosenthal MA and Oratz R: Phase II clinical trial of recombinant alpha $2 \mathrm{~b}$ interferon and 13 cis retinoic acid in patients with metastatic melanoma. Am J Clin Oncol 21: 352-354, 1998.

37. Sondak VK, Liu PY, Flaherty LE, et al: A phase II evaluation of all-trans-retinoic acid plus interferon alfa-2a in stage IV melanoma: a Southwest Oncology Group study. Cancer J Sci Am 5: 41-47, 1999.

38. Epping MT and Bernards R: A causal role for the human tumor antigen preferentially expressed antigen of melanoma in cancer. Cancer Res 66: 10639-10642, 2006.

39. Li L, Giannopoulos K, Reinhardt P, et al: Immunotherapy for patients with acute myeloid leukemia using autologous dendritic cells generated from leukemic blasts. Int J Oncol 28: 855-861, 2006 . 